\title{
CFD modelling of wall-particle interactions under turbulent flow conditions
}

\author{
M. Mollagee \\ Department of Chemical Engineering, University of Johannesburg, \\ South Africa
}

\begin{abstract}
The effects of particle roughness and the impact it has on the development of turbulence in non-Newtonian slurry flow remains difficult to predict. The common analytical tools used take only the viscous characteristics of the slurry into account. Homogenous solid-liquid suspensions are often described using different continuum models. Evidence suggests that these models may be inadequate due to the presence of solid particles sharply influencing velocity gradients. Experimental work was conducted using homogenous non-Newtonian slurries. Comparisons were made of wall-particle interactions experienced for slurries with different representative particle sizes. This was subsequently modeled using the FLUENT Computational Fluid Dynamics software to validate these findings. This paper documents these findings and presents a comparison between the experimental and the computational model.
\end{abstract}

Keywords: non-Newtonian slurries, experimental versus computational methods, viscous sub-layer, wall turbulence, wall-particle interactions.

\section{Introduction}

Turbulent flow of non-Newtonian fluids continues to attract the attention of researchers from fields as diverse as physics, hydraulics and engineering. The flow dynamics differs markedly when compared to the behaviour under laminar conditions. Reliable prediction has presented complex theoretical as well as practical problems, both from the point of view of the fundamental physics of the phenomenon as well as in engineering practice. The principle dilemma is whether the turbulent headloss can be predicted from the rheology of the slurry alone, or whether other properties also play a role. In the case of mine tailings where slurries are regarded as stratified flow systems, accurate prediction can be challenging from two perspectives: 
a. The slurry has to be characterised as non-Newtonian, which is often not the case;

b. When operating within the economically profitable transition zone just prior to the onset of turbulence, most modelling tools are of an empirical nature. Treating a slurry as a continuum aggravates the problem.

\section{Literature review}

\subsection{Non-Newtonian turbulence models}

There are many different approaches to turbulence modelling for non-Newtonian slurries in pipes. Some of these models are presented below.

Wilson and Thomas [1] developed a model that produced an analysis of turbulent flow based on enhanced micro-scale viscosity effects. The viscous sublayer is predicted to thicken by an area ratio $\left(A_{r}\right)$. This ratio is defined as the relation between the non-Newtonian and assumed Newtonian rheograms under identical shear conditions. The area ratio is given by:

$$
A_{r}=2\left[\frac{1+\frac{\tau_{y}}{\tau_{0}}}{1+n}\right]
$$

The velocity distribution is given by:

$$
u^{+}=\frac{u}{V_{*}}=2.5 \ln \left[\frac{\rho V_{*}}{\mu}\right]+5.5+11.6\left(A_{r}-1\right)-2.5 \ln \left(A_{r}\right)
$$

Treating slurries as a continuum works well in the laminar regime where flow is dominated by viscous effects. Slatter [2] proposed a model that accounts for the effects of particle roughness. The model suggests that if the solid particles are of the same order of magnitude as the viscous sub-layer the continuum approximation is compromised in the wall region. A new Reynolds number was developed to predict the onset of turbulence. Schlichting [3] was the first to suggest that a roughness Reynolds number can be used to determine the various regions of turbulent flow in pipes. It is formulated as follows:

$$
\operatorname{Re}_{r}=\frac{8 \rho V_{*}^{2}}{\tau_{y}+K\left[\frac{8 V_{*}}{d_{x}}\right]^{n}}
$$

If $\operatorname{Re}_{\mathrm{r}}<3.32$, smooth wall turbulence exists and the mean velocity is given by:

$$
\frac{V}{V_{*}}=2.5 \ln \left(\frac{R}{d_{x}}\right)+2.5 \ln \mathrm{Re}_{r}+1.75
$$

If Rer $>3.32$, fully developed rough wall turbulent flow exists and the mean velocity is given by: 


$$
\frac{V}{V_{*}}=2.5 \ln \left(\frac{R}{d_{x}}\right)+1.75
$$

Chilton and Stainsby [4] concluded that the friction factor could be directly correlated to the Reynolds number provided that the viscosity at the wall is properly evaluated. Many researchers have supported this conclusion.

\subsection{Evidence of particle roughness}

Park et al. [5] used laser techniques to investigate the turbulent structure of nonNewtonian slurries. Higher intensities were observed in the wall region when compared with that of air. Pokryvalio and Grozberg [6] used electro-diffusion methods to measure the velocity profile of Bentonite clay suspensions and a similar phenomenon had been reported. Mun [7] used fine and coarse ilmenite and coal suspensions and compared this to 24 other correlations in the literature. A variation in turbulence intensity as a function of particle size was found.

\subsection{The CFD model}

The commercial CFD software package FLUENT 6.1.22 was used for solving the governing set of equations. The discretization equations along with initial boundary conditions were solved using the segregated solver to obtain a numerical solution. Simulating flows in the near-wall region is common in many applications. This is due to the presence of the viscous sub-layer where molecular diffusion and viscous dissipation dominates. The sub-layer has an influence on the overall development of turbulence. Adequate numerical resolution requires a very fine mesh due to the thinness of this layer. The highReynolds number (HR) models do not in themselves provide resolution of the viscous sub-layer. The boundary conditions in the case of HR models are represented by wall functions with a limited application. It does however significantly save on computational time. White and Christoph [8] and Huang et al. [9] proposed a low-Reynolds (LR) $\mathrm{k}-\varepsilon$ model to address this problem.

\subsubsection{Available models}

Turbulent flows in general are significantly affected by the presence of a stationary wall. The mean velocity field is influenced by the no-slip condition that exists at the wall. Viscous damping tends to reduce the tangential velocity fluctuations while kinematic blocking reduces the normal fluctuations. Towards the outer part of the near-wall region the turbulence is rapidly augmented by the production of turbulent kinetic energy due to the large gradients in mean velocity. The solution variables in the wall region have large gradients and momentum and other scalar quantities occur vigorously. Accurate representation of flow in the near-wall region determines successful predictions of wallbounded turbulent flows. The $k-\varepsilon$, Reynolds Stress, and Large Eddy Simulation models are all primarily valid for turbulent flow in the core of the flow domain under study. The Spalart-Allmaras and $k$ - $\omega$ models are designed for application throughout the boundary layer provided that the wall mesh resolution is sufficient. 


\subsubsection{The near-wall region}

The near-wall region can be sub-divided into three distinctive layers. The "viscous sub-layer" is the innermost layer. The molecular viscosity plays a dominant role in momentum and heat or mass transfer. Turbulence dominates in the outer "fully turbulent layer". There is an "interim region" between the viscous sub-layer and the fully turbulent layer where the effects of molecular viscosity and turbulence are equally important.

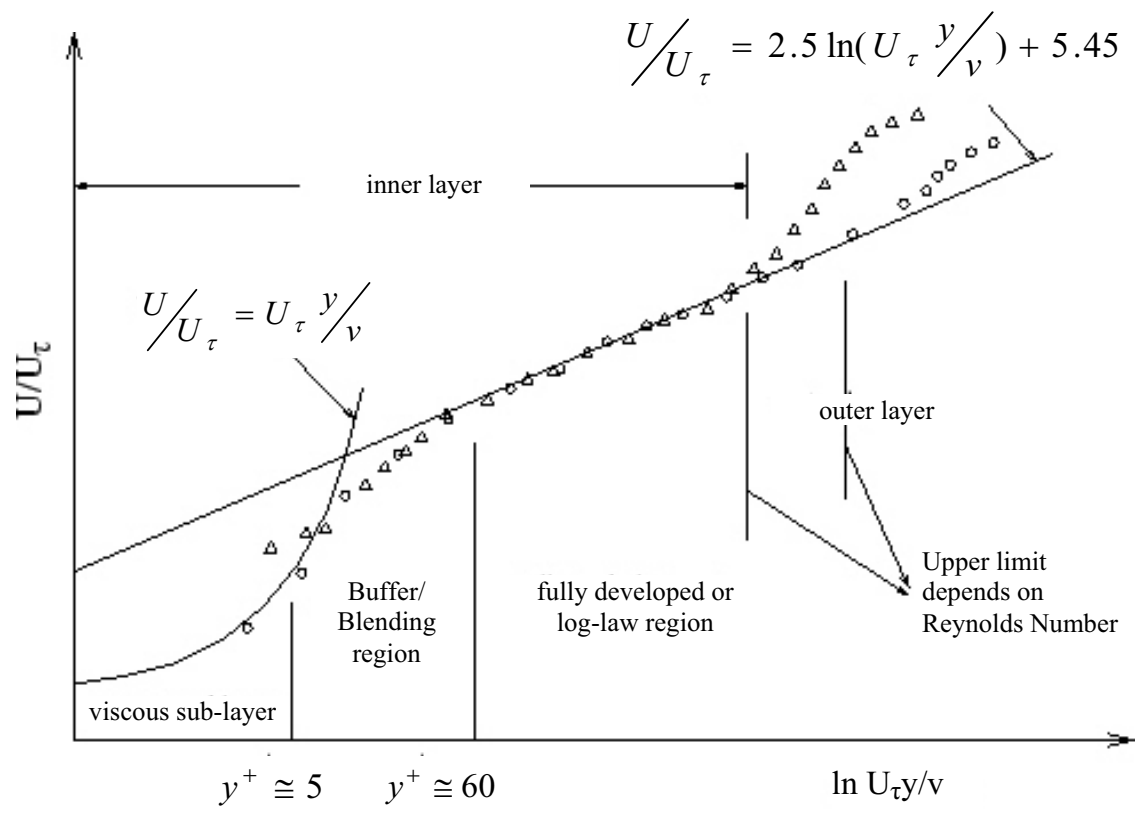

Figure 1: Sub-divisions of the near-wall region.

Traditionally there are two approaches to modelling the near-wall region. In the first approach the viscosity-affected inner region (viscous sub-layer and buffer layer) is not resolved. Semi-empirical formulae (wall functions) are used to bridge the region between the wall and the fully turbulent region. The use of wall functions averts the need to modify the turbulence models to account for the presence of the wall. In HR flows this approach saves computational resources in the near-wall region, where solution variables change rapidly and no resolution is required. It is inadequate in situations where the low-Reynoldsnumber effects are pervasive and the hypotheses underlying wall functions cease to be valid. Such situations require near-wall models that are valid in the viscosity-affected region and solvable all the way to the wall.

\subsubsection{Two-layer model for enhanced wall treatment}

A near-wall formulation that can be used with coarse meshes (wall-function meshes) as well as fine meshes (LR meshes) is required. In addition, excessive error should not be incurred for intermediate meshes that are too fine for the 
near-wall cell centroid to lie in the fully turbulent region, but also too coarse to properly resolve the sub-layer. In FLUENT the wall region is resolved all the way to the viscous sub-layer. The two-layer approach is an integral part of the enhanced wall treatment and is used to specify both $\varepsilon$ and the turbulent viscosity in the near-wall cells. In this approach the whole domain is subdivided into a viscosity-affected region and a fully turbulent region. A wall-distance-based turbulent Reynolds number, $\mathrm{Re}_{y}$, demarcates the two regions:

$$
\operatorname{Re}_{y}=\frac{\rho y \sqrt{k}}{\mu}
$$

where $y$ is the normal distance from the wall at the cell centres.

In the fully developed region the standard k- $\varepsilon$ is employed. In the near-wall region the one-equation model of Wolfstein [10] is used. The turbulent viscosity $\mu_{\mathrm{t}}$ is re-calculated as follows:

$$
\mu_{t, 2-\text { layer }}=\rho C_{\mu} \ell_{\mu} \sqrt{k}
$$

The length scale is found from Chen and Patel [11]:

$$
\ell_{\mu}=y c_{\ell}\left(1-e^{-\operatorname{Re}_{y} / A_{\mu}}\right)
$$

The two-layer formulation is used as part of the enhanced wall treatment where it is smoothly blended with the $\mathrm{HR} \mu_{t}$ definition from the outer region as proposed by Jongen [12]:

$$
\mu_{t, \text { enhanced }}=\lambda_{\varepsilon} \mu_{t}+\left(1-\lambda_{\varepsilon}\right) \mu_{t, t w o-l a y e r}
$$

$\mu_{\mathrm{t}}$ is the HR definition for the k- $\varepsilon$ model. A blending function $\lambda_{\varepsilon}$ is chosen such that it is equal to unity far from the walls and zero close to the wall:

$$
\lambda_{\varepsilon}=\frac{1}{2}\left[1+\tanh \left(\frac{\operatorname{Re}_{y}-\operatorname{Re}^{*} y}{A}\right)\right]
$$

The constant $A$ determines the width of the blending function. A width is defined such that the value of $\lambda_{\varepsilon}$ will be within $1 \%$ of its far-field value:

$$
A=\frac{\left|\Delta \operatorname{Re}_{y}\right|}{\tanh (0.98)}
$$

$\Delta \operatorname{Re}_{\mathrm{y}}$ would typically be assigned a value of between $5 \%$ and $20 \%$ of $\Delta \operatorname{Re}^{*} \mathrm{y}$. Blending is aimed at preventing the solution convergence from being impeded when the k- $\varepsilon$ solution does not match the two-layer solution.

\section{Experimental}

\subsection{Apparatus}

The experimental work was conducted as part of the slurry flow research programme at the Cape Peninsula University of Technology (Cape Town, South Africa). A laboratory-scale tube viscometer with pipes of 4 different diameters 
was used to generate the experimental data. Mollagee [13] provides more of the construction-related detail.

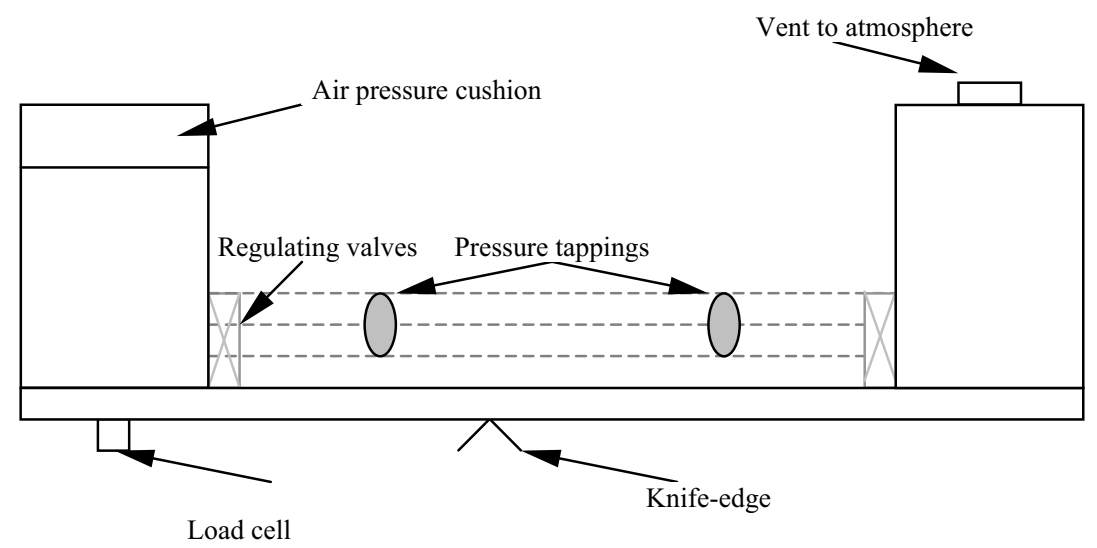

Figure 2: $\quad$ Layout of tube viscometer.

\subsection{Mixtures}

Sand was introduced into a pure kaolin mixture to gradually increase the particle sizes. Tests were conducted at different relative densities to establish the amount of kaolin required to fully suspend the sand. A volumetric concentration of $6 \%$ was used as the baseline test set.

Table 1: $\quad$ Summary of slurry properties.

\begin{tabular}{|l|l|l|l|l|l|c|}
\hline Test Set & \multicolumn{1}{|c|}{ Slurry } & $\mathrm{Cv}(\%)$ & $\tau_{\mathrm{y}}[\mathrm{Pa}]$ & $\mathrm{K}$ & $\mathrm{n}$ & $\rho_{\mathrm{s}}$ \\
\hline $\mathrm{K} \_1$ & Pure Kaolin & 6 & 5.077 & 0.0043 & 0.719 & 1091.8 \\
\hline $\mathrm{K} \_2$ & Kaolin/sand & 9 & 7.522 & 0.014 & 0.845 & 1139.1 \\
\hline K 3 & Kaolin/sand & 12 & 9.848 & 0.018 & 0.831 & 1200.8 \\
\hline K 4 Kaolin/sand & 15 & 11.55 & 0.031 & 0.786 & 1244.5 \\
\hline
\end{tabular}

\subsection{Numerical computation}

The differential equations governing turbulent flow in a straight pipe could be written in tensor form in the master Cartesian coordinate system as:

Conservation equation for mass:

$$
\nabla \cdot(\rho \vec{u})=0
$$

Conservation equation for momentum:

$$
\nabla \cdot(\vec{u} \vec{u})=-\nabla p+\nabla \cdot\left[\mu\left(\nabla \vec{u}+\nabla \vec{u}^{\Gamma}\right)\right\rfloor+\rho \vec{g}+\vec{F}
$$

The governing equations were solved with a control volume finite element method. The FLUENT/UNS code [14] has been used as the numerical solver. 


\subsubsection{Grid system}

An unstructured non-uniform grid system was used to discretize the governing equations. The convection term in the governing equations was modeled with a bounded first-order upwind scheme. A typical hexahedral element was applied for the three-dimensional grid. The pattern used for this paper consists of 27 nodes, with information stored on the vertex, mid-edge mid-face and center nodes.

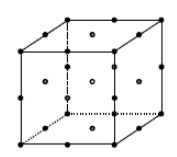

Figure 3: $\quad$ 27-node pattern.

\subsubsection{Solution strategy}

A second-order upwind discretization scheme was used for the momentum equations while a first-order scheme was used for the turbulent kinetic energy and turbulent dissipation energy. These schemes ensured in general satisfactory accuracy, stability and convergence. Other strategies employed were reduction of under-relaxation factors for momentum, turbulent kinetic energy and turbulent dissipation energy to resolve any non-linearity.

\section{Results and discussion}

The various turbulent models under review are presented in Table 2, and Figures 4 and 5. The laminar data obtained during the rheological characterisation procedure was obtained through integration across the length of the pipe to furnish the parameters for the Power Law Model. This was found to agree with the literature in that intermediate values for fluid consistency $(K)$ were obtained.

The Yield Pseudoplastic model produced the lowest average percentage error (APE) when compared to the other rheological models. The Wilson and Thomas (W \& T) model produced the highest error with the Slatter model performing the best with an APE of 9.84\%. The Chilton and Stainsby (C \& S) and the CFD model performed similarly. This could partially be attributed to the numerical one-dimensional finite difference model they had used [15]. The CFD model consistently produced APE's in the range of 11.96 (lowest) and 21.00 (highest). The dispersion of particles due to turbulence was predicted using the stochastic tracking model or the particle cloud model. In both models the particles have no direct impact on the generation or dissipation of turbulence in the continuous phase. This was of concern and could be the reason behind the higher APE's. The modelling in the wall region needs to be further investigated.

Figure 4 demonstrates the effect of increasing the concentration and coarse fraction of the slurry by the metered addition of amounts of sand to a pure kaolin mixture. This was done to increase the representative particle size. The effects of increased concentration can be seen when examined in conjunction with the Slatter model. 
Table 2: $\quad$ Tabulated average percentage error by rheological model.

\begin{tabular}{|l|r|r|r|r|}
\hline \multicolumn{1}{|c|}{ Slurry } & W \& T & \multicolumn{1}{c|}{ Slatter } & C \& S & \multicolumn{1}{c|}{ CFD } \\
\hline K1_5mm & 19.59 & 8.96 & 8.08 & 9.47 \\
\hline K1_13mm & 18.82 & 9.51 & 10.60 & 11.96 \\
\hline K1_28mm & 10.04 & 7.19 & 18.94 & 16.28 \\
\hline K1_46mm & 6.29 & 11.71 & 20.25 & 21.00 \\
\hline K2_5mm & 17.00 & 8.31 & 3.16 & 16.89 \\
\hline K2_13mm & 19.40 & 9.65 & 7.93 & 15.24 \\
\hline K2_28mm & 26.59 & 19.42 & 9.36 & 14.77 \\
\hline K2_46mm & 11.87 & 6.49 & 6.57 & 10.55 \\
\hline K3_5mm & 20.80 & 16.39 & 7.00 & 13.90 \\
\hline K3_13mm & 12.97 & 8.63 & 4.92 & 17.21 \\
\hline K3_28mm & 17.77 & 11.94 & 7.11 & 13.84 \\
\hline K3_46mm & 10.57 & 7.23 & 3.82 & 8.45 \\
\hline K4_5mm & 12.69 & 11.62 & 14.02 & 13.33 \\
\hline K4_13mm & 8.68 & 5.51 & 8.50 & 9.66 \\
\hline K4_28mm & 13.88 & 7.28 & 16.23 & 14.57 \\
\hline K4_46mm & 6.81 & 7.53 & 18.90 & 17.14 \\
\hline AVERAGE & $\mathbf{1 4 . 6 1}$ & $\mathbf{9 . 8 4}$ & $\mathbf{1 0 . 3 4}$ & $\mathbf{1 4 . 0 2}$ \\
\hline
\end{tabular}

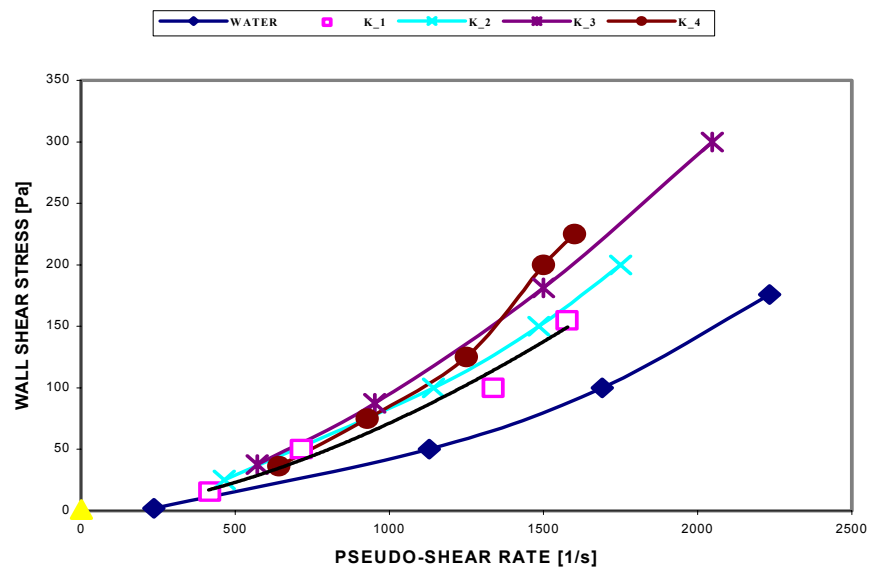

Figure 4: Effect of increasing concentration for $46 \mathrm{~mm}$ pipe.

Figure 5 gives us a snapshot of the various turbulence models under discussion. The Slatter (APE-9.51\%) and C \& S models (APE-10.60\%) were virtually super-imposable for the $13 \mathrm{~mm}$ pipe. The FLUENT model overpredicted at low shear rates and under-predicted at higher wall shear stresses. 
Although the APE's were only 1\% higher (11.96\%), this was only due to the net compensating effect across the data spectrum. This erratic scatter can be attributed to modelling inconsistencies in the wall region where particle roughness effects has been proven to exist.

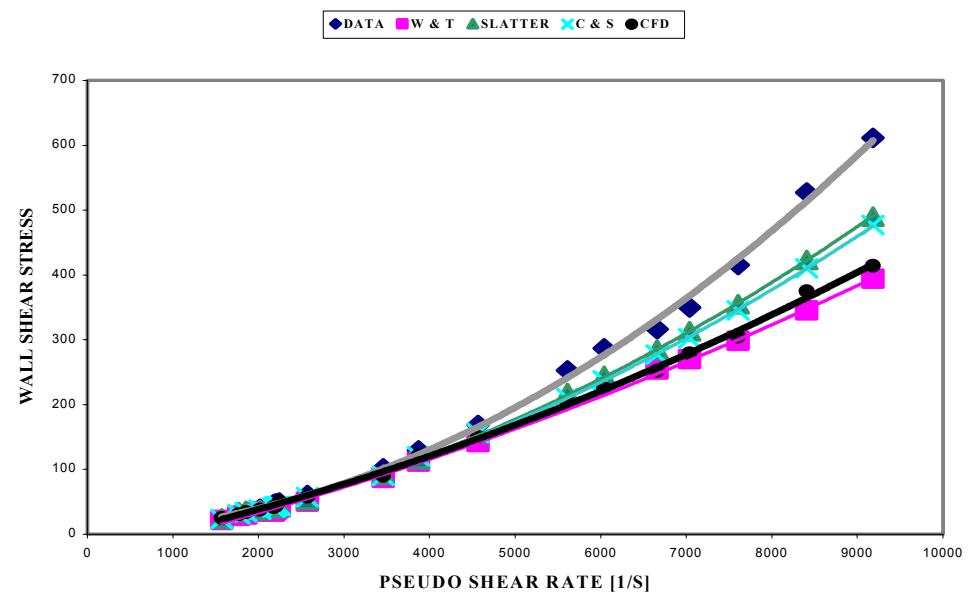

Figure 5: $\quad$ Pseudo shear diagram for $\mathrm{K}_{-} 1$ mixture $-13 \mathrm{~mm}$ pipe.

\section{Conclusion}

The phenomenon of turbulence for non-Newtonian slurry flow in pipelines was examined in this paper. Computational fluid dynamics software (FLUENT 6.1) was used to perform the numerical calculations. The numerical profile was compared to the particle roughness model of Slatter, the viscous sub-layer model of Wilson and Thomas and the Blasius-type numerical model of Chilton and Stainsby. The agreement between experiment and simulation was found to be within a $15 \%$ range of error and can be regarded as satisfactory. The two areas requiring more in-depth investigation are the near-wall region and the particle tracking techniques used in this study.

\section{Acknowledegment}

The author wishes to thank the University of Johannesburg for providing the opportunity to proceed with this work.

\section{References}

[1] Wilson K C and Thomas A D (1985), A new analysis of the turbulent flow of non-Newtonian fluids, Can. J. Chem. Eng., 63, 539-546.

[2] Slatter P T (1994), Transitional and turbulent flow of non-Newtonian slurries in pipes, PhD Thesis, University of Cape Town. 
[3] Schlichting H (1960), Chapter XX in Boundary Layer Theory, $4^{\text {th }}$ edition, McGraw-Hill, New York.

[4] Chilton R A and Stainsby R (1996), Prediction of pressure losses in turbulent non-Newtonian flows, Proc. Of $13^{\text {th }}$ International Conference on Slurry Handling and Pipeline Transport BHR Group HYDROTRANSPORT 13, 21-40.

[5] Park J T, Mannheimer R J, Grimley T A, Morrow T B (1989), Pipeflow measurements of a transparent non-Newtonian slurry, Journal of Fluids Engineering, Vol. 111, 331-336.

[6] Pokryvalio N A and Grozberg Y G (1995), Investigation of the structure of turbulent wall flow of clay suspensions in channels with electrodiffusion methods, Proc. $8^{\text {th }}$ International Conf. On Transport and Sedimentation of Solid Particles, Prague, Czech Republic.

[7] Mun R (1988), Turbulent pipe flow of yield stress fluids, M. Eng. Sci. Thesis, University of Melbourne.

[8] White F and Christoph G, A Simple New Analysis of Compressible Turbulent Skin Friction Under Arbitrary Conditions. Technical Report AFFDL-TR-70-133, February 1971.

[9] Huang P, Bradshaw P and Coakley T, Skin Friction and Velocity Profile Family for Compressible Turbulent Boundary Layers, AIAA Journal, 31(9), 1600-1604, September 1993.

[10] Wolfstein M, The Velocity and Temperature Distribution of OneDimensional Flow with Turbulence Augmentation and Pressure Gradient. Int. J. Heat Mass Transfer, 12:301-318, 1969.

[11] Chen H C and Patel V C, Near-Wall Turbulence Models for Complex Flows Including Separation. AIAA Journal, 26(6), 641-648, 1988.

[12] Jongen T, Simulation and Modeling of Turbulent Incompressible Flows. PhD thesis, EPF Lausanne, Lausanne, Switzerland, 1992.

[13] Mollagee M M, Particle Roughness Turbulence in the Balanced Beam Tube Viscometer, MTech Thesis, CPUT, Cape Town, South Africa, 1998.

[14] FLUENT 6.1. Users Guide, 2003.

[15] Stainsby R, Chilton R A and Thompson S C, Prediction of Head Losses in non-Newtonian Sludge Pipelines using CFD, Proc. $2^{\text {nd }}$ CFDS Int. User Conf., Pittsburgh, USA, 259-272, 1994. 